J. Korean Math. Soc. 47 (2010), No. 4, pp. 819-830

DOI 10.4134/JKMS.2010.47.4.819

\title{
PIERCE STALKS OF EXCHANGE RINGS
}

\author{
HuANYin Chen
}

\begin{abstract}
We prove, in this article, that a ring $R$ is a stable exchange ring if and only if so are all its Pierce stalks. If every Pierce stalks of $R$ is artinian, then $1_{R}=u+v$ with $u, v \in U(R)$ if and only if for any $a \in R$, there exist $u, v \in U(R)$ such that $a=u+v$. Furthermore, there exists $u \in U(R)$ such that $1_{R} \pm u \in U(R)$ if and only if for any $a \in R$, there exists $u \in U(R)$ such that $a \pm u \in U(R)$. We will give analogues to normal exchange rings. The root properties of such exchange rings are also obtained.
\end{abstract}

\section{Introduction}

A ring $R$ is called an exchange ring if for every right $R$-module $A$ and any two decompositions $A=M \oplus N=\bigoplus_{i \in I} A_{i}$, where $M_{R} \simeq R_{R}$ and $I$ is a finite index set, there exist submodules $A_{i}^{\prime} \subseteq A_{i}$ such that $A=M \oplus\left(\bigoplus_{i \in I} A_{i}^{\prime}\right)$. It is well known that regular rings, $\pi$-regular rings, unit $C^{*}$-algebras of real rank zero, semiperfect rings, left or right continuous rings and clean rings are all exchange rings (cf. [1]).

Let $S(R)$ be the nonempty set of all the proper ideals of a ring $R$ generated by central idempotents. By the Zorn's Lemma, $S(R)$ contains maximal elements. If $P$ is a maximal element of the set $S(R)$, then the factor ring $R / P$ is called a Pierce stalk of $R$. In [11, Proposition 32.1], Tuganbaev characterized exchange rings by their Pierce stalks. He proved that a ring $R$ is an exchange ring if and only if so are all its Pierce stalks. The present paper is such an attempt in this direction.

Recall that a ring $R$ is a stable ring provided that $a R+b R=R$ with $a, b \in R$ implies that there exists $y \in R$ such that $a+b y \in U(R)$ (cf. [6-7] and [10]). In this article, we will characterize stable exchange rings by their Pierce stalks. The third section studies rings with artinian Pierce stalks. These will give a new class of exchange rings. If every Pierce stalks of $R$ is artinian, then $1_{R}=u+v$ with $u, v \in U(R)$ if and only if for any $a \in R$, there exist $u, v \in U(R)$ such that $a=u+v$, i.e., $R$ is a $(s, 2)$ ring. Furthermore, there exists $u \in U(R)$ such that $1_{R} \pm u \in U(R)$ if and only if for any $a \in R$, there exists $u \in U(R)$ such that

Received October 2, 2008.

2000 Mathematics Subject Classification. 16E50, 19 U99.

Key words and phrases. exchange ring, Pierce stalk, stable ring. 
$a \pm u \in U(R)$. For any $n \times n(n \geq 2)$ matrix $A$ over such ring, it is shown that there exists $U \in G L_{n}(R)$ such that $A \pm U \in G L_{n}(R)$. We will extend these results to normal exchange rings. The root properties of such exchange rings are also obtained.

Throughout this paper, all rings are associative with identity and all modules are unital right $R$-modules. $A \lesssim^{\oplus} B$ means that $A$ is isomorphic to a direct summand of $B$ and $n A$ denotes the $n$ copies of $A$.

\section{Stable exchange rings}

It is well known that if $\operatorname{End}_{R}(A)$ has stable range one, then $A \oplus B \cong A \oplus$ $C$ implies that $B \cong C$ for any right $R$-modules. Stable range one plays an important rule in non-stable $K$-theory.

Theorem 2.1. Let $R$ be a ring. Then the following are equivalent:

(1) $R$ is a stable exchange ring.

(2) Every Pierce stalk of $R$ is a stable exchange ring.

Proof. (1) $\Rightarrow(2)$ Clearly, every Pierce stalk of $R$ is a stable ring. In view of [11, Proposition 32.1], it is an exchange ring.

$(2) \Rightarrow(1)$ By $[11$, Proposition 32.1], $R$ is an exchange ring. Given $a R+$ $b R=R$, then we can find some $x, y \in R$ such that $a x+b y=1$. Let $f\left(X_{1}, X_{2}, Y_{1}, Y_{2}\right)=1-\left(X_{1}+X_{2} Y_{1}\right) Y_{2}, g\left(X_{1}, X_{2}, Y_{1}, Y_{2}\right)=1-Y_{2}\left(X_{1}+X_{2} Y_{1}\right)$ be the polynomials in noncommutative indeterminate $X_{1}, X_{2}, Y_{1}$ and $Y_{2}$. Let $R / P$ be an arbitrary Pierce stalk of $R$. Then $\overline{a x}+\bar{b} \bar{y}=\overline{1}$ in $R / P$. By assumption, there exists a $\bar{z} \in R / P$ such that $\bar{a}+\bar{b} \bar{y} z \in U(R / P)$. Thus, we have $u \in R$ such that $\overline{u(a+b y z)}=\overline{1}=\overline{(a+b y z)} \bar{u}$, and so $f(\bar{a}, \bar{b}, \overline{y z}, \bar{u})=\overline{0}$ and $g(\bar{a}, \bar{b}, \overline{y z}, \bar{u})=\overline{0}$. In view of [11, Lemma 11.4], there exist $t, w \in R$ such that $f(a, b, t, w)=0$ and $g(a, b, t, w)=0$. That is, $(a+b t) w=1_{R}=w(a+b t)$. This implies that $a+b t \in U(R)$, and therefore we complete the proof.

A ring $R$ is a unit-regular ring in case for any $a \in R$, there exists $u \in U(R)$ such that $a=a u a$.

Corollary 2.2. Let $R$ be a ring. Then the following are equivalent:

(1) $R$ is a unit-regular ring.

(2) Every Pierce stalk of $R$ is a unit-regular ring.

Proof. (1) $\Rightarrow(2)$ is obvious.

$(2) \Rightarrow(1)$ In view of [11, Remark 11.7], $R$ is a regular ring. By virtue of Theorem 2.1, $R$ is a stable ring. Thus, $R$ is unit-regular from [8, Proposition 4.12 .

Lemma 2.3. Let $I, J$ and $K$ be ideals of a stable exchange ring $R$ such that $K=I J$. Let $A$ and $B$ be finitely generated projective right $R$-modules.

(a) If $A / A I \cong B / B I$ and $A / A J \cong B / B J$, then $A / A K \cong B / B K$.

(b) If $A / A I \lesssim B / B I$ and $A / A J \lesssim \oplus B / B J$, then $A / A K \lesssim \oplus B / B K$. 
Proof. (a) Suppose that $A / A I \cong B / B I$ and $A / A J \cong B / B J$. As in the proof of [1, Proposition 1.4], we can find right $R$-modules $A_{1}, A_{2}, B_{1}, B_{2}$ such that $A \cong A_{1} \oplus B_{1}$ and $B \cong A_{2} \oplus B_{2}$ with $A_{1} \cong A_{2}, B_{1}=B_{1} I, B_{2}=B_{2} I$. Clearly, $B_{1} J=B_{1} K$ and $B_{2} J=B_{2} K$. Thus, $A_{1} / A_{1} J \oplus B_{1} / B_{1} K \cong A / A J \cong B / B J \cong$ $A_{2} / A_{2} J \oplus B_{2} / B_{2} K$ with $A_{1} / A_{1} J \cong A_{2} / A_{2} J$. Since $R$ is a stable ring, so is $R / J$. Hence, we deduce that $B_{1} / B_{1} K \cong B_{2} / B_{2} K$. Therefore $A / A K \cong A_{1} / A_{1} K \oplus$ $B_{1} / B_{1} K \cong A_{2} / A_{2} K \oplus B_{2} / B_{2} K \cong A / A K$.

(b) Suppose that $A / A I \lesssim^{\oplus} B / B I$ and $A / A J \lesssim^{\oplus} B / B J$. As in the proof of [1, Proposition 1.4], we can find right $R$-modules $A_{1}, A_{2}, B_{1}, B_{2}$ such that $A \cong$ $A_{1} \oplus B_{1}$ and $B \cong A_{2} \oplus B_{2}$ with $A_{1} \cong A_{2}, B_{1}=B_{1} I$. Thus, $A_{1} / A_{1} J \oplus B_{1} / B_{1} J \cong$ $A / A J \lesssim B / B J \cong A_{2} / A_{2} J \oplus B_{2} / B_{2} J$ with $A_{1} / A_{1} J \cong A_{2} / A_{2} J$. As a result, $B_{1} / B_{1} J \lesssim \oplus B_{2} / B_{2} J$. Hence, there exists a split epimorphism $\varphi: B_{2} / B_{2} J \rightarrow$ $B_{1} / B_{1} J$. Clearly, there exists an epimorphism $\psi: B_{2} / B_{2} K \rightarrow B_{2} / B_{2} J$ given by $\psi\left(b+B_{2} K\right)=b+B_{2} J$ for any $b+B_{2} K \in B_{2} / B_{2} K$. As $B_{1} J=B_{1} K$, we can find a split epimorphism $\varphi \psi: B_{2} / B_{2} K \rightarrow B_{1} / B_{1} K$. This implies that $B_{1} / B_{1} K \lesssim^{\oplus} B_{2} / B_{2} K$. So we conclude that $A / A K \cong A_{1} / A_{1} K \oplus B_{1} / B_{1} K \lesssim^{\oplus}$ $A_{2} / A_{2} K \oplus B_{2} / B_{2} K \cong A / A K$.

Theorem 2.4. Let $A$ and $B$ be finitely generated projective right modules over a stable exchange ring $R$.

(a) If $A / A P \cong B / B P$ for all Pierce stalks $R / P$, then $A \cong B$.

(b) If $A / A P \lesssim^{\oplus} B / B P$ for all Pierce stalks $R / P$, then $A \lesssim^{\oplus} B$.

Proof. (a) Since $A$ and $B$ are finitely generated projective right modules over a stable exchange ring $R$, we can find $E=E^{2}, F=F^{2} \in M_{n}(R)$ such that $A \cong E(n R)$ and $B \cong F(n R)$. Hence, $\bar{E}(n(R / P)) \cong \bar{F}(n(R / P))$. Assume that $A \neq B$. Then $E(n R) \neq F(n R)$. Let

$$
\Omega=\{P \mid P \text { is an ideal of } R \text { such that } \bar{E}(n(R / P)) \neq \bar{F}(n(R / P))\} \text {. }
$$

Clearly, $0 \in \Omega$; hence, $\Omega \neq \emptyset$. Given $P_{1} \subseteq P_{2} \subseteq \cdots \subseteq P_{n} \subseteq \cdots$ in $\Omega$, we will prove that $Q:=\bigcup_{i=1}^{\infty} P_{i} \in \Omega$. If not, then $\bar{E}(n(R / Q)) \cong \bar{F}(n(R / Q))$; hence, $\bar{E} M_{n}(R / Q) \cong \bar{F} M_{n}(R / Q)$. Thus, we can find some $X, Y \in M_{n}(R)$ such that $\bar{E}=\overline{X Y}, \bar{F}=\overline{Y X}, \bar{E}=\overline{E F E}$ and $\bar{F}=\overline{F E F}$. That is, $E \equiv X Y, F \equiv$ $Y X, X \equiv X Y X, Y \equiv Y X Y\left(\bmod M_{n}(Q)\right)$. As a result, we can find an ideal $P \in \Omega$ such that $E \equiv X Y, F \equiv Y X, X \equiv X Y X, Y \equiv Y X Y\left(\bmod M_{n}(P)\right)$. It follows that $\bar{E} M_{n}(R / P) \cong \bar{F} M_{n}(R / P)$, and so $\bar{E}(n(R / P)) \cong \bar{F}(n(R / P))$. This gives a contradiction. In other words, $\Omega$ is inductive. By using Zorn's Lemma, we can find an ideal $M$ of $R$, which is maximal in $\Omega$. Since $A / A M \neq$ $B / B M, R / M$ is not a Pierce stalk of $R$. In view of [11, Remark 11.1], there exists a central idempotent $e \in R$ such that $M+e R$ and $M+(1-e) R$ are both proper ideals of $R$. By the maximal of $M$, we see that $A / A(M+e R) \cong$ $B / B(M+e R)$ and $A / A(M+(1-e) R) \cong B / B(M+(1-e) R)$. Clearly, $(M+e R)(M+(1-e) R)=M=(M+(1-e) R)(M+e R)$. According to Lemma $2.3, A / A M \cong B / B M$, a contradiction. This shows that $A \cong B$. 
(b) is proved in the same manner.

Corollary 2.5. Let $A$ be a finitely generated projective right module over a stable exchange ring $R$, and let $n$ be a positive integer. If $A / A P$ can be generated by $n$ elements for all Pierce stalks $R / P$, then $A$ can be generated by $n$ elements.

Proof. Let $R / P$ be a Pierce stalk of $R$. Since $A / A P$ is a projective right $R$ module and is generated by $n$ elements, $A / A P \lesssim \oplus(n R) /(n R) P$. According to Theorem 2.4, we see that $A \lesssim \oplus R$. Thus, $A$ can be generated by $n$ elements.

Recall that an exchange ring $R$ is normal provided that every idempotent in $R$ is central. Let $A$ be a finitely generated projective right module over a normal exchange ring $R$. In view of [11, Proposition 32.2], all Pierce stalks of a normal exchange ring are local. Thus $A / A P$ is a finitely generated free $R$-module; hence, $\operatorname{dim}_{R}(A / A P)$ is well defined.

Corollary 2.6. Let $A$ and $B$ be finitely generated projective right modules over a normal exchange ring $R$.

(a) If $\operatorname{dim}_{R / P}(A / A P)=\operatorname{dim}_{R / P}(B / B P)$ for all Pierce stalks $R / P$, then $A \cong B$.

(b) If $\operatorname{dim}_{R / P}(A / A P) \leq B / B P$ for all Pierce stalks $R / P$, then $A \lesssim^{\oplus} B$.

Proof. (a) For any Pierce stalk $R / P$ of $R, R / P$ is a local ring by [11, Proposition 32.2]. If $\operatorname{dim}_{R / P}(A / A P)=B / B P$, then $A / A P \cong B / B P$. In view of Theorem 2.4, we conclude that $A \cong B$.

(b) is proved in the same manner.

\section{Artinian Pierce stalks}

In this section, we introduce a new class of exchange rings. We say that $R$ is a ring with artinian Pierce stalks provided that whose Pierce stalks are all right artinian rings.

Lemma 3.1. Let $R$ be a ring with artinian Pierce stalks. Then $R$ is a stable exchange ring.

Proof. Since every artinian ring is a stable ring, we complete the proof by Lemma 2.1.

It is well known that every exchange ring with artinian primitive factors has root property. For the rings with artinian Pierce stalks, we can derive a similar result.

Theorem 3.2. Let $R$ be a ring with artinian Pierce stalks. Let $A$ and $B$ be finitely generated projective right $R$-module, and let $n$ be a positive integer.

(a) If $n A \cong n B$, then $A \cong B$.

(b) If $n A \lesssim^{\oplus} n B$, then $A \lesssim^{\oplus} B$. 
Proof. (a) Assume that $n A \cong n B$. If $R / P$ is a Pierce stalk of $R$, then $n(A / A P) \cong n(B / B P)$. Since $R / P$ is an artinian ring, $A / A P \cong B / B P$. Therefore we conclude that $A \cong B$ from Theorem 2.4.

(b) is proved in the same manner.

Corollary 3.3. Let $R$ and $S$ be rings with artinian Pierce stalks. If $M_{n}(R) \cong$ $M_{n}(S)$ for some positive integer $n$, then $R \cong S$.

Proof. Let $T=M_{n}(R)$. Then we can find idempotents $e, f \in T$ such that $R \cong e T e$ and $S \cong f T f$. In addition, $n(e T) \cong n(f T)$. In view of Lemma 3.1, $T$ is an exchange ring. Let $T / I$ be a Pierce stalk of $T$. Then we can find some central idempotents $E_{1}, \ldots, E_{n} \in M_{n}(R)$ such that $I=T E_{1} T+\cdots+T E_{n} T$. One easily checks that each $E_{i}=\operatorname{diag}\left(e_{i}, \ldots, e_{i}\right) \in M_{i}(R)$ for some central idempotent $e_{i} \in R$. Clearly, there exists an ideal $P$ of $R$ such that $I=M_{n}(P)$. In addition, $P=R e_{1} R+\cdots+R e_{n} R$. If $R / P$ is not a Pierce stalk of $R$, then we can find a central idempotent $e \in R$ such that $P \varsubsetneqq P+e R$ is a proper ideal of $R$. Let $F=\operatorname{diag}(e, \ldots, e) \in M_{n}(R)$. Then $F \in M_{n}(R)$ is a central idempotent matrix. Clearly, $I \varsubsetneqq T E_{1} T+\cdots+T E_{n} T+T F T \varsubsetneqq R$. This shows that $T / I$ is not a Pierce stalk of $T$, a contradiction. Thus, $R / P$ is a Pierce stalk of $R$. By assumption, $R / P$ is artinian. Since $T / I \cong M_{n}(R / P)$, we see that $T / I$ is artinian. In other words, $T$ is exchange rings with artinian Pierce stalks. According to Theorem 3.2, we deduce that $e T \cong f T$, and therefore $R \cong S$.

Let $R$ be an exchange ring with artinian primitive factors. In [4, Theorem 3], the author proved that the following are equivalent: (1) Every element in $R$ is a sum of two units. (2) There exist units $\alpha, \beta$ in $R$ such that $1=\alpha+\beta$. Now we extend this result to rings with artinian Pierce stalks.

Theorem 3.4. Let $R$ be a ring with artinian Pierce stalks. Then the following are equivalent:

(1) $1_{R}=u+v$ with $u, v \in U(R)$.

(2) For any $a \in R$, there exist $u, v \in U(R)$ such that $a=u+v$.

(3) $a R+b R=R$ with $a, R \in R$ implies that there exists $u \in U(R)$ such that $a+b u \in U(R)$.

Proof. (1) $\Rightarrow(3)$ Assume that $a R+b R=R$ with $a, R \in R$. Let $f\left(X_{1}, X_{2}, Y_{1}, Y_{2}\right)$ $=1-\left(X_{1}+X_{2} Y_{1}\right) Y_{2}, g\left(X_{1}, X_{2}, Y_{1}, Y_{2}\right)=1-Y_{2}\left(X_{1}+X_{2} Y_{1}\right), h\left(Y_{1}, Y_{3}\right)=1-Y_{1} Y_{3}$ and $k\left(Y_{1}, Y_{3}\right)=1-Y_{3} Y_{1}$ be the polynomials in noncommutative indeterminate $X_{1}, X_{2}, Y_{1}, Y_{2}$ and $Y_{3}$. Let $R / P$ be a Pierce stalk of $R$. By assumption, we have $\overline{1_{R}}=\bar{u}+\bar{v}$ in $R / P$. Since $R / P$ is artinian, it is an exchange ring with artinian primitive factors. In view of [4, Theorem 3], we can find a unit $\bar{u} \in U(R / P)$ such that $\bar{a}+\overline{b u} \in U(R / P)$. Thus, there exist some $\bar{v}, \bar{w} \in R / P$ such that $(\bar{a}+\overline{b u}) \bar{w}=\overline{1_{R}}=\bar{w}(\bar{a}+\overline{b u})$ and $\overline{u v}=\overline{1_{R}}=\overline{v u}$. That is, $f(\bar{a}, \bar{b}, \bar{u}, \bar{w})=$ $\overline{0}, g(\bar{a}, \bar{b}, \bar{u}, \bar{w})=\overline{0}, h(\bar{u}, \bar{v})=\overline{0}$ and $k(\bar{u}, \bar{v})=\overline{0}$. According to [11, Lemma 11.4], 
we can find some $s, t, c \in R$ such that $f(a, b, s, t)=0, g(a, b, s, t)=0, h(t, c)=0$ and $k(c, t)=0$. This implies that $a+b s \in U(R)$ and $u \in U(R)$, as required.

$(3) \Rightarrow(2)$ For any $a \in R$, we have $a \times 0+1 \times 1=1$, hence, $a R+R=R$. By assumption, we can find $u \in U(R)$ such that $a+u \in U(R)$, as desired.

$(2) \Rightarrow(1)$ is trivial.

Corollary 3.5. Let $R$ be a ring with artinian Pierce stalks. If $2 \in U(R)$, then for any $A \in M_{n}(R)$, there exists $U \in G L_{n}(R)$ such that $A+U \in G L_{n}(R)$.

Proof. Let $A \in M_{n}(R)(n \geq 1)$. As in the proof of Corollary 3.3, $M_{n}(R)$ is an exchange ring with artinian Pierce stalks. By virtue of Theorem 3.4, we complete the proof.

Let $R=\mathbb{Z} / 3 \mathbb{Z}$. Then there exists $u \in U(R)$ such that $a+u \in U(R)$. But there is no any $u \in U(R)$ such that $a \pm u \in U(R)$. Now we extend Theorem 3.4 as follows.

Theorem 3.6. Let $R$ be a ring with artinian Pierce stalks. Then the following are equivalent:

(1) There exists $u \in U(R)$ such that $1_{R} \pm u \in U(R)$.

(2) For any $a \in R$, there exists $u \in U(R)$ such that $a \pm u \in U(R)$.

Proof. (1) $\Rightarrow(2)$ Let $a \in R$. Let

$$
\begin{aligned}
f\left(X, Y_{1}, Y_{2}\right) & =1-\left(X+Y_{1}\right) Y_{2}, g\left(X, Y_{1}, Y_{2}\right)=1-Y_{2}\left(X+Y_{1}\right), \\
h\left(X, Y_{1}, Y_{3}\right) & =1-Y_{3}\left(X-Y_{1}\right), k\left(X, Y_{1}, Y_{3}\right)=1-\left(X-Y_{1}\right) Y_{3}, \\
l\left(Y_{1}, Y_{4}\right) & =1-Y_{1} Y_{4}
\end{aligned}
$$

and $p\left(Y_{1}, Y_{4}\right)=1-Y_{4} Y_{1}$ be the polynomials in noncommutative indeterminate $X_{1}, X_{2}, Y_{1}, Y_{2}, Y_{3}$ and $Y_{4}$. Let $R / P$ be a Pierce stalk of $R$. By hypothesis, we have $\overline{1_{R}} \pm \bar{u} \in U(R / P)$. Since $R / P$ is artinian, $(R / P) / J(R / P)$ is semisimple. Thus, we can find some division rings $D_{1}, \ldots, D_{n}$ such that $(R / P) / J(R / P) \cong$ $\bigoplus_{i=1}^{n} M_{m_{i}}\left(D_{i}\right)$. Let $A \in M_{m_{i}}\left(D_{i}\right)$.

If $m_{i}=1$, by assumption, $1_{D_{i}} \pm u_{i} \in U\left(D_{i}\right)$ for some $u_{i} \in U\left(D_{i}\right)$. If $A=0$, then $A \pm 1_{D_{i}} \in U\left(D_{i}\right)$. If $A \neq 0$, then $A \pm A u_{i} \in U\left(D_{i}\right)$.

If $m_{i} \geq 2$, there exist $U, V \in G L_{m_{i}}\left(D_{i}\right)$ such that

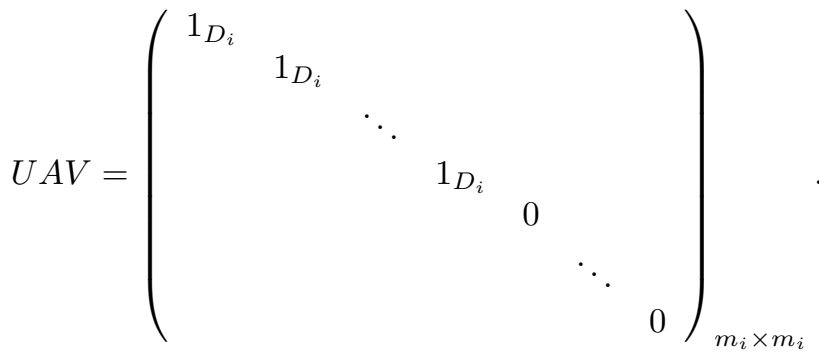


Assume that $m_{i}\left(m_{i} \geq 2\right)$ is an even number. Let

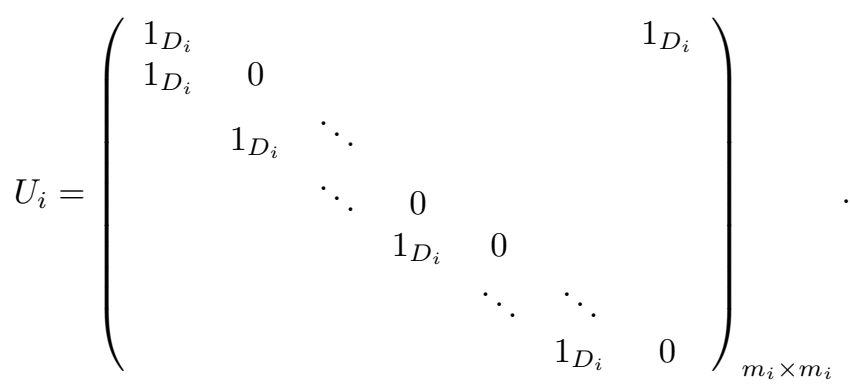

Then $U_{i} \in G L_{m_{i}}\left(D_{i}\right)$. Clearly,

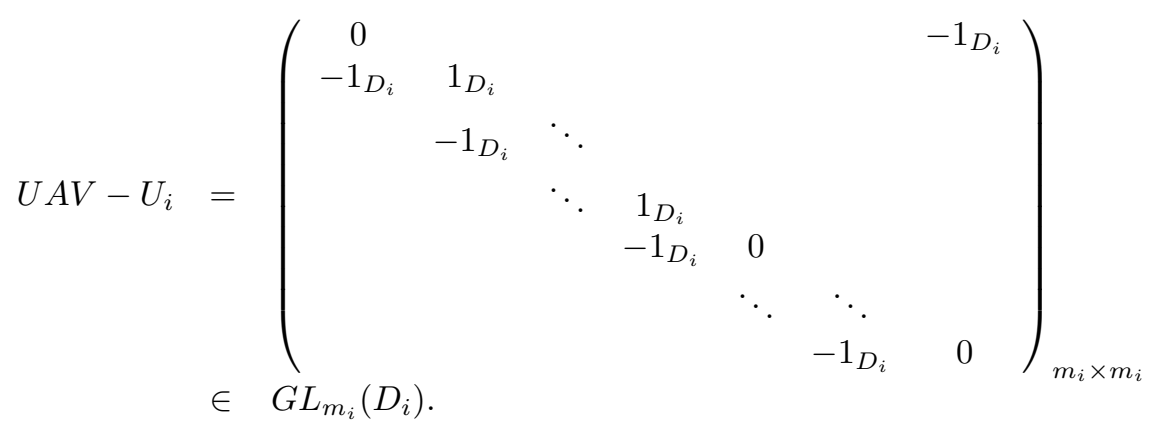

On the other hand,

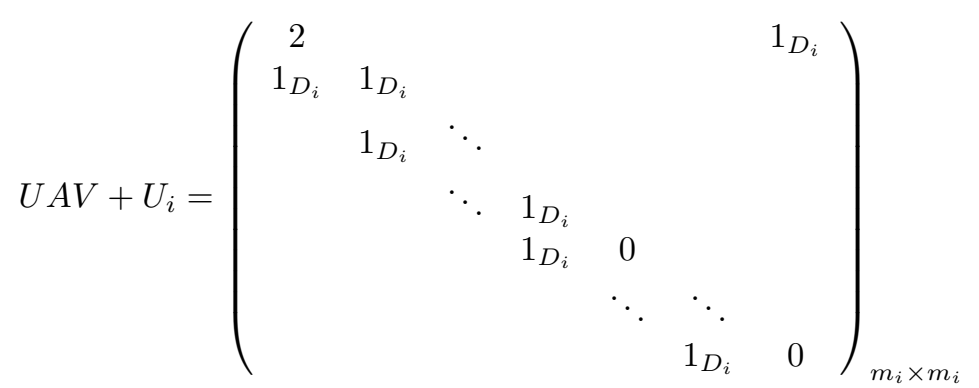

or

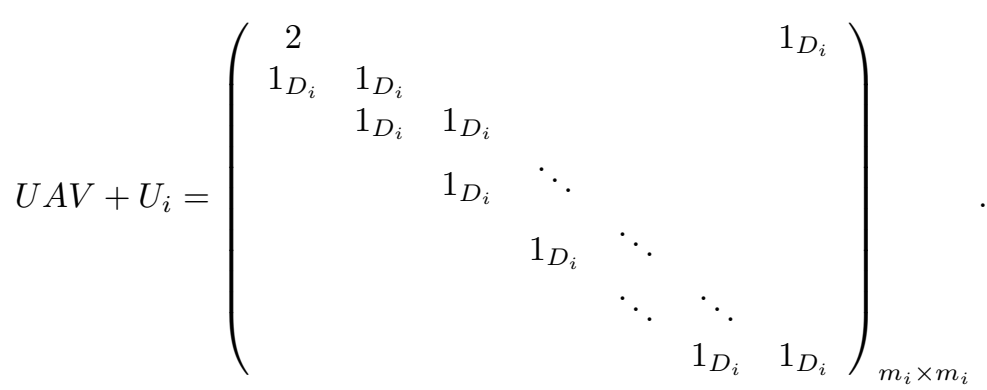

As $m_{i}$ is an even number, one easily checks that $U A V+U_{i} \in G L_{m_{i}}\left(D_{i}\right)$. 
Assume that $m_{i}\left(m_{i} \geq 2\right)$ is an odd number. Let

$$
U_{i}=\left(\begin{array}{ccccccc}
1_{D_{i}} & & & & & & 1_{D_{i}} \\
-1_{D_{i}} & 0 & & & & & \\
& 1_{D_{i}} & \ddots & & & & \\
& & \ddots & 0 & & & \\
& & & 1_{D_{i}} & 0 & & \\
& & & & \ddots & \ddots & \\
& & & & & 1_{D_{i}} & 0
\end{array}\right)_{m_{i} \times m_{i}}
$$

Then

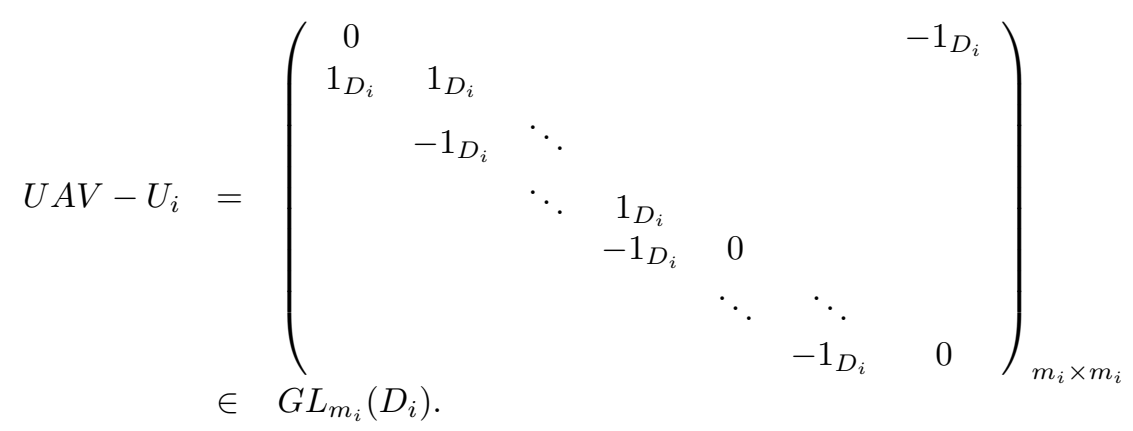

On the other hand,

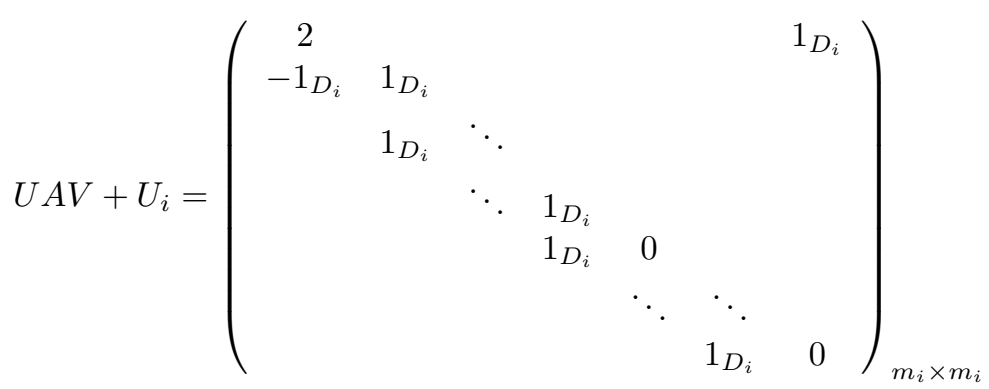

or

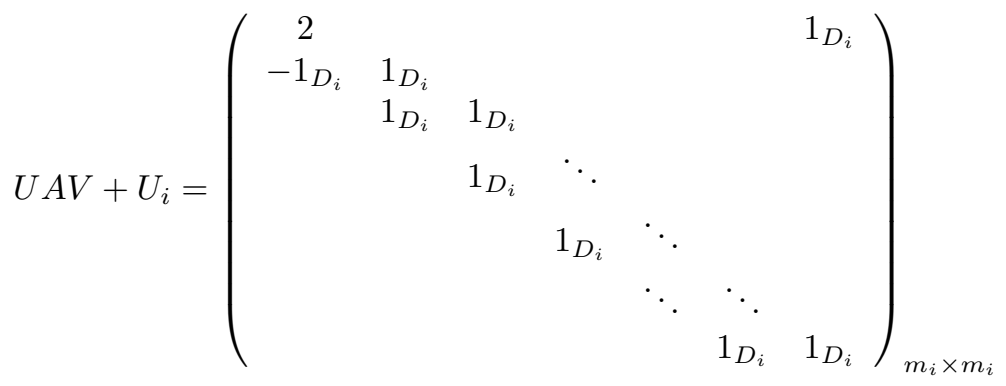


As $m_{i}$ is an odd number, one easily checks that $U A V+U_{i} \in G L_{m_{i}}\left(D_{i}\right)$. In any case, we can find $U_{i} \in G L_{m_{i}}\left(D_{i}\right)$ such that $U A V \pm U_{i} \in G L_{m_{i}}\left(D_{i}\right)$, and so $A \pm U^{-1} U_{i} V^{-1} \in G L_{m_{i}}\left(D_{i}\right)$.

By the consideration above, we can find a $\bar{u} \in U((R / P) / J(R / P))$ such that $\overline{(a+P)} \pm \bar{u} \in U((R / P) / J(R / P))$. Clearly, $u \in U(R / P)$. Furthermore, we show that $(a+P) \pm u \in U(R / P)$. Hence, there exist some $v, s, t \in R / P$ such that $(\bar{a}+u) s=\overline{1_{R}}=s(\bar{a}+u),(\bar{a}-u) t=\overline{1_{R}}=t(\bar{a}-u)$ and $u v=\overline{1_{R}}=v u$. That is, $f(\bar{a}, u, s)=\overline{0}, g(\bar{a}, u, s)=\overline{0}, h(\bar{a}, u, t)=\overline{0}, k(\bar{a}, u, t)=\overline{0}, l(u, v)=\overline{0}$ and $p(u, v)=\overline{0}$. According to [11, Lemma 11.4], we can find some $b, c, d, q \in R$ such that $f(\bar{a}, b, c)=0, g(\bar{a}, b, c)=0, h(\bar{a}, b, d)=0, k(\bar{a}, b, d)=0, l(b, q)=0$ and $p(b, q)=0$. This implies that $a \pm b \in U(R)$ and $b \in U(R)$, as desired.

$(2) \Rightarrow(1)$ is obvious.

Corollary 3.7. Let $R$ be a ring with artinian Pierce stalks. Then for any $A \in M_{n}(R)(n \geq 2)$, there exists $U \in G L_{n}(R)$ such that $A \pm U \in G L_{n}(R)$.

Proof. Let $A \in M_{n}(R)$. As in the proof of Theorem 3.6, we can find $U \in$ $G L_{n}(R)$ such that $\operatorname{diag}(1, \ldots, 1)_{n \times n} \pm U \in G L_{n}(R)$. By virtue of Theorem 3.6, there exists $U \in G L_{n}(R)$ such that $A \pm U \in G L_{n}(R)$.

Corollary 3.8. Let $R$ be a ring with artinian Pierce stalks, and let $n$ be a positive integer. If $2,3 \in U(R)$, then for any $A \in M_{n}(R)$, there exists $U \in$ $G L_{n}(R)$ such that $A \pm U \in G L_{n}(R)$.

Proof. Clearly, $1_{R}=2^{-1}+2^{-1}$ and $1_{R}=-2^{-1}+3 \times 2^{-1}$. By virtue of Theorem 3.6 and Corollary 3.7, we complete the proof.

Recall that a ring $R$ is a semilocal ring provided that $R / J(R)$ is right artinian. Let $R$ be a semilocal ring with $2,3 \in U(R)$. Then for any $A \in M_{n}(R)$, there exists $U \in G L_{n}(R)$ such that $A \pm U \in G L_{n}(R)$. Applying Corollary 3.8 to $R / J(R)$, we are done.

Example 3.9. Let $D=\{0, e, a, b\}$ be a set. Define operations by the following tables:

\begin{tabular}{c|cccc}
+ & 0 & $e$ & $a$ & $b$ \\
\hline 0 & 0 & $e$ & $a$ & $b$ \\
$e$ & $e$ & 0 & $b$ & $a$ \\
$a$ & $a$ & $b$ & 0 & $e$ \\
$b$ & $b$ & $a$ & $e$ & 0
\end{tabular}

\begin{tabular}{c|cccc}
$\times$ & 0 & $e$ & $a$ & $b$ \\
\hline 0 & 0 & 0 & 0 & 0 \\
$e$ & 0 & $e$ & $a$ & $b$ \\
$a$ & 0 & $a$ & $b$ & $e$ \\
$b$ & 0 & $b$ & $e$ & $a$
\end{tabular}

Then $D$ is a field. Let $R=M_{n}(D), A \in M_{n}(D)$. Clearly, $R$ is a ring with artinian Pierce stalks. It is easy to verify that $e \pm a=b \in U(R)$. By virtue of Theorem 3.6 and Corollary 3.7, we can find $U \in G L_{n}(R)$ such that $A \pm U \in$ $G L_{n}(R)$. In this case, $2 \notin U(R)$.

Recall that a ring $R$ is clean if each element of $R$ can be written as the sum of an idempotent and a unit. As every artinian ring is a clean ring, so is every ring with artinian Pierce stalks. 


\section{Normal exchange rings}

In [5, Theorem 10], the author provides an element-wise characterization of normal exchange rings, namely $R$ is an exchange ring with all idempotents central if and only if for any $x \in R$ there exist a central idempotent $e$ and a unit $u$ such that $x=e+u$. Now we extend this result as follows.

Theorem 4.1. Let $R$ be a ring. Then the following are equivalent:

(1) $R$ is a normal exchange ring.

(2) $a R+b R=R$ with $a, R \in R$ implies that there exists a central idempotent $e \in R$ such that $a+b e \in U(R)$.

Proof. (1) $\Rightarrow(2)$ Assume that $a R+b R=R$ with $a, b \in R$ and $c \in R$. Let $f\left(X_{1}, X_{2}, Y_{1}, Y_{2}\right)=1-\left(X_{1}+X_{2} Y_{1}\right) Y_{2}, g\left(X_{1}, X_{2}, Y_{1}, Y_{2}\right)=1-Y_{2}\left(X_{1}+\right.$ $\left.X_{2} Y_{1}\right), h\left(X_{3}, Y_{2}\right)=X_{3} Y_{2}-Y_{2} X_{3}$ and $k\left(Y_{1}\right)=Y_{1}-Y_{1}^{2}$ be the polynomials in noncommutative indeterminate $X_{1}, X_{2}, X_{3}, Y_{1}$ and $Y_{2}$. Let $R / P$ be an arbitrary Pierce stalk of $R$. Then we can find some $x, y \in R$ such that $\overline{a x}+\bar{b} \bar{y}=\overline{1}$ in $R / P$. In view of [11, Proposition 32.2], $R / P$ is a local ring. If $\bar{a} \notin J(R / P)$, then $\bar{a}+\bar{b} \times \overline{0} \in U(R / P)$. If $\bar{a} \in J(R / P)$, it follows from $\bar{a}(\bar{x}-\bar{y})+(\bar{a}+\bar{b}) \bar{y}=\overline{1}$ that $\bar{a}+\bar{b} \times \overline{1} \in U(R / P)$. In any case, we can find an idempotent $f \in R / P$ such that $\bar{a}+\bar{b} f \in U(R / P)$ and $f \bar{c}=\bar{c} f$. Thus, we have a $w \in R / P$ such that $(\bar{a}+\bar{b} f) w=\overline{1}=w(\bar{a}+\bar{b} f)$. This means that $f(\bar{a}, \bar{b}, f, w)=\overline{0}, g(\bar{a}, \bar{b}, f, w)=$ $\overline{0}, h(\bar{c}, f)=\overline{0}$ and $k(f)=\overline{0}$. In view of [11, Lemma 11.4], there exist $e, d \in R$ such that $f(a, b, e, d)=0, g(a, b, e, d)=0, h(c, e)=0$ and $k(e)=0$. This implies that we can find a central idempotent $e \in R$ such that $a+b e \in U(R)$, as asserted.

$(2) \Rightarrow(1)$ For any $x \in R$, we have $x \times 0+(-1) \times(-1)=1$, and so $x R+$ $(-1) R=R$. By assumption, we can find a central idempotent $e \in R$ such that $x-e \in U(R)$, and therefore we complete the proof by [11, Proposition 32.2].

Corollary 4.2. Let $R$ be a ring. Then the following are equivalent:

(1) $R$ is a normal exchange ring.

(2) For any $x, y \in R$, there exists a central idempotent $e \in R$ such that $1+x(y-e) \in U(R)$.

Proof. $(1) \Rightarrow(2)$ For any $x, y \in R$, we have $(-x) y+(1+x y)=1$; hence, $(1+x y) R+(-x) R=R$. By assumption, we have a central idempotent $e \in R$ such that $(1+x y)+(-x) e \in U(R)$, as required.

$(2) \Rightarrow(1)$ Given any $x \in R$, we can find a central idempotent $e \in R$ such that $1+1 \times(-x-e) \in U(R)$. Let $u=1+1 \times(-x-e)$. Then $x=(1-e)+(-u)$ is the sum of a central idempotent and a unit. Therefore the proof is true by [11, Proposition 32.2].

Theorem 4.3. Let $R$ be a normal exchange ring. Then the following are equivalent:

(1) There exists $u \in U(R)$ such that $1_{R} \pm u \in U(R)$. 
(2) For any $a \in R$, there exists $u \in U(R)$ such that $a \pm u \in U(R)$.

Proof. $(2) \Rightarrow(1)$ is obvious.

$(1) \Rightarrow(2)$ Let $a \in R$. Let $f\left(X, Y_{1}, Y_{2}\right)=1-\left(X+Y_{1}\right) Y_{2}, g\left(X, Y_{1}, Y_{2}\right)=$ $1-Y_{2}\left(X+Y_{1}\right), h\left(X, Y_{1}, Y_{3}\right)=1-Y_{3}\left(X-Y_{1}\right), k\left(X, Y_{1}, Y_{3}\right)=1-(X-$ $\left.Y_{1}\right) Y_{3}, l\left(Y_{1}, Y_{4}\right)=1-Y_{1} Y_{4}$ and $p\left(Y_{1}, Y_{4}\right)=1-Y_{4} Y_{1}$ be the polynomials in noncommutative indeterminate $X_{1}, X_{2}, Y_{1}, Y_{2}, Y_{3}$ and $Y_{4}$. Let $R / P$ be a Pierce stalk of $R$. By hypothesis, we have $\overline{1_{R}} \pm \bar{u} \in U(R / P)$. In view of [11, Proposition 32.2], $R / P$ is a local ring, $(R / P) / J(R / P)$ is a division ring. As in the proof in Theorem 3.6, we can find $u \in U(R / P)$ such that $\bar{a} \pm u \in U(R / P)$. Hence, there exist some $v, s, t \in R / P$ such that $(\bar{a}+u) s=\overline{1_{R}}=s(\bar{a}+u),(\bar{a}-u) t=\overline{1_{R}}=$ $t(\bar{a}-u)$ and $u v=\overline{1_{R}}=v u$. That is, $f(\bar{a}, u, s)=\overline{0}, g(\bar{a}, u, s)=\overline{0}, h(\bar{a}, u, t)=$ $\overline{0}, k(\bar{a}, u, t)=\overline{0}, l(u, v)=\overline{0}$ and $p(u, v)=\overline{0}$. According to [11, Lemma 11.4], there are some $b, c, d, q \in R$ such that $f(\bar{a}, b, c)=0, g(\bar{a}, b, c)=0, h(\bar{a}, b, d)=$ $0, k(\bar{a}, b, d)=0, l(b, q)=0$ and $p(b, q)=0$. Thus, $a \pm b \in U(R)$ and $b \in U(R)$, as required.

A ring $R$ is a right (left) quasi-duo if every maximal right (left) ideal is two-sided.

Corollary 4.4. Let $R$ be a right (left) quasi-duo exchange ring. Then the following are equivalent:

(1) There exists $u \in U(R)$ such that $1_{R} \pm u \in U(R)$.

(2) For any $a \in R$, there exists $u \in U(R)$ such that $a \pm u \in U(R)$.

Proof. $(2) \Rightarrow(1)$ is clear.

$(1) \Rightarrow(2)$ Since $R$ is a right (left) quasi-duo exchange ring, by [12, Lemma 2.3], $R / J(R)$ is a normal exchange ring. For any $a \in R$, there exists $\bar{u} \in U(R / J(R))$ such that $\bar{a} \pm \bar{u} \in U(R / J(R))$. As every unit lifts modulo the Jacobson radical, we easily obtain the result.

Corollary 4.5. Let $R$ be a right (left) quasi-duo exchange ring. If $2,3 \in U(R)$, then for any $x \in R$, there exists $u \in U(R)$ such that $x \pm u \in U(R)$.

Proof. If $2,3 \in U(R)$, then $1_{R}=2^{-1}+2^{-1}$ and $1_{R}=-2^{-1}+3 \times 2^{-1}$. Therefore the result follows by Corollary 4.4 .

Theorem 4.6. Let $R$ be a normal exchange ring. Then for any $A \in M_{n}(R)$ $(n \geq 2)$, there exists $U \in G L_{n}(R)$ such that $A \pm U \in G L_{n}(R)$.

Proof. Let $A \in M_{n}(R)$. Let $f\left(X, Y_{1}, Y_{2}\right)=1-\left(X+Y_{1}\right) Y_{2}, g\left(X, Y_{1}, Y_{2}\right)=$ $1-Y_{2}\left(X+Y_{1}\right), h\left(X, Y_{1}, Y_{3}\right)=1-Y_{3}\left(X-Y_{1}\right), k\left(X, Y_{1}, Y_{3}\right)=1-(X-$ $\left.Y_{1}\right) Y_{3}, l\left(Y_{1}, Y_{4}\right)=1-Y_{1} Y_{4}$ and $p\left(Y_{1}, Y_{4}\right)=1-Y_{4} Y_{1}$ be the polynomials in noncommutative indeterminate $X_{1}, X_{2}, Y_{1}, Y_{2}, Y_{3}$ and $Y_{4}$. Let $M_{n}(R) / I$ be a Pierce stalk of $M_{n}(R)$. As in the proof of Corollary 3.3, we can find an ideal $P$ of $R$ such that $I=M_{n}(P)$ and $R / P$ is a Pierce stalk of $R$. By [11, Proposition 32.2], $R / P$ is a local ring. Hence $(R / P) / J(R / P)$ is a division ring. Let $D=(R / P) / J(R / P)$. Then $\left(M_{n}(R) / I\right) / J\left(M_{n}(R) / I\right) \cong M_{n}(D)$. 
As in the proof of Theorem 3.6, we can find $V \in U\left(M_{n}(R) / I\right)$ such that $\bar{A} \pm V \in U\left(M_{n}(R) / I\right)$. Thus, we have some $u, s, t \in M_{n}(R) / I$ such that $f(\bar{a}, u, s)=\overline{0}, g(\bar{a}, u, s)=\overline{0}, h(\bar{a}, u, t)=\overline{0}, k(\bar{a}, u, t)=\overline{0}, l(u, v)=\overline{0}$ and $p(u, v)=\overline{0}$. According to [11, Lemma 11.4], we have some $U, c, d, q \in M_{n}(R)$ such that $f(\bar{a}, U, c)=0, g(\bar{a}, U, c)=0, h(\bar{a}, U, d)=0, k(\bar{a}, U, d)=0, l(U, q)=0$ and $p(U, q)=0$. This implies that $A \pm U, U \in G L_{n}(R)$, and we are done.

Corollary 4.7. Let $R$ be a right (left) quasi-duo exchange ring. Then for any $A \in M_{n}(R)(n \geq 2)$, there exists $U \in G L_{n}(R)$ such that $A \pm U \in G L_{n}(R)$.

Proof. Let $A \in M_{n}(R)$. Since $R$ is a right (left) quasi-duo exchange ring, by [12, Lemma 2.3], $R / J(R)$ is a normal exchange ring. In view of Theorem 4.6, there exists $\bar{U} \in G L_{n}(R / J(R))$ such that $\bar{A} \pm \bar{U} \in G L_{n}(R / J(R))$. As in the proof of Corollary 4.4, we see that $U \in G L_{n}(R)$ and $A \pm U \in G L_{n}(R)$, as asserted.

Corollary 4.8. Let $R$ be an abelian regular ring. Then for any $A \in M_{n}(R)$ $(n \geq 2)$, there exists $U \in G L_{n}(R)$ such that $A \pm U \in G L_{n}(R)$.

Proof. Since every abelian regular ring is a right (left) quasi-duo exchange ring, we complete the proof by Corollary 4.7 .

\section{References}

[1] P. Ara, K. R. Goodearl, K. C. O'Meara, and E. Pardo, Separative cancellation for projective modules over exchange rings, Israel J. Math. 105 (1998), 105-137.

[2] D. G. Burkholder, Azumaya rings, Pierce stalks and central ideal algebras, Comm. Algebra 17 (1989), no. 1, 103-113.

[3] _ Modules with local Pierce stalks, J. Algebra 145 (1992), no. 2, 339-348.

[4] H. Chen, Exchange rings with Artinian primitive factors, Algebr. Represent. Theory 2 (1999), no. 2, 201-207.

[5] On exchange rings with all idempotents central, Algebra Colloq. 6 (1999), no. $1,45-50$.

[6] - On stable range conditions, Comm. Algebra 28 (2000), no. 8, 3913-3924.

[7] H. Chen, Invertible matrices over weakly stable rings, J. Korean Math. Soc. 46 (2009), no. 2, 257-269.

[8] K. R. Goodearl, Von Neumann Regular Rings, Pitman, London-San Francisco-Melbourne, 1979; 2nd ed., Krieger, Malabar, Fl., 1991.

[9] C. Huh, N. K. Kim, and Y. Lee, On exchange rings with primitive factor rings Artinian, Comm. Algebra 28 (2000), no. 10, 4989-4993.

[10] T. Y. Lam, A crash course on stable range, cancellation, substitution and exchange, J. Algebra Appl. 3 (2004), no. 3, 301-343.

[11] A. A. Tuganbaev, Rings Close to Regular, Kluwer Academic Publishers, Dordrecht, Boston, London, 2002.

[12] H. P. Yu, On quasi-duo rings, Glasgow Math. J. 37 (1995), no. 1, 21-31.

Department of Mathematics

HANGZHOU NORMAL UNIVERSITY

Hangzhou 310036, P. R. China

E-mail address: huanyinchen@yahoo.cn 